\title{
EFEITO DO EXTRATO AQUOSO DE PLANTAS SOBRE A BROCA DA BANANEIRA Cosmopolites sordidus Germar (Coleoptera: Curculionidae)
}

PAVARINI, Ronaldo ${ }^{1}$

ANSANTE, Thiago Felipe ${ }^{2}$

CACERES, Danilo Pereira ${ }^{2}$

Recebido em: 2009. 12. 28

Aprovado em: 2010.12.12

ISSUE DOI: $10.3738 / 1982.2278-341$

\begin{abstract}
RESUMO: Com o objetivo de avaliar a porcentagem de repelência de extratos aquosos de plantas sobre o adulto da broca da bananeira (Cosmopolites sordidus), testou-se os extratos de diferentes estruturas das seguintes plantas: Azadirachta indica, Pimenta pseudocaryophyllus, Trichilia pallens, Trichilia pallida, Ricinus communis e Ocimum minimum. O experimento foi conduzido em condições de laboratório com 10 tratamentos, provenientes dos diferentes extratos e 5 repetições, sendo cada repetição formada por um grupo de 10 insetos adultos provenientes de bananal comercial. Os maiores valores em relação a repelência foram observados quando se utilizou extrato obtido a partir de sementes de Azadirachta indica e folhas de Pimenta pseudocaryophyllus, com 48 e $24 \%$ de repelência, respectivamente, sendo estes valores estatisticamente diferentes entre si. A porcentagem de repelência dos extratos aquosos obtidos a partir de folhas de Azadirachta indica, Trichilia pallens e Trichilia pallida e ramos de Trichilia pallens foi de 11,54 \%, não diferindo estatisticamente dos extratos aquosos obtidos a partir de ramos de Pimenta pseudocaryophyllus e Ocimum minimum e folhas de Ricinus communis e Ocimum minimum. De acordo com os resultados obtidos, conclui-se que os diferentes extratos de plantas apresentaram atividade de repelência sobre os adultos da broca da bananeira, mas, no entanto, as maiores porcentagens de foram observadas quando se utilizou extrato obtido a partir de sementes de Azadirachta indica e folhas de Pimenta pseudocaryophyllus.
\end{abstract}

Palavras-chave: Plantas inseticidas. Resistência de insetos. Manejo de pragas.

\section{EFFECT OF AQUEOUS EXTRACT OF PLANTS ON THE BANANA WEEVIL Cosmopolites sordidus Germar (Coleoptera: Curculionidae)}

\begin{abstract}
SUMMARY: In order to evaluate repellency percentage of aqueous extracts of plants on the adult of banana weevil (Cosmopolites sordidus), it was tested the extracts of different structures of the following plants: Azadirachta indica, Pimenta pseudocaryophyllus, Trichilia pallens, Trichilia pallida, Ricinus communis and Ocimum minimum. The experiment was conducted under laboratory conditions with 10 treatments, obtained by the different extracts, and 5 replicates, each one consisting of a group of 10 adult insects from commercial banana field. The highest values of repellency were observed when using extracts derived from seeds of Azadirachta indica and leaves of Pimenta pseudocaryophyllus, 48 and $24 \%$ of repellence, respectively. These values were statistically different between them. The percentage of repellency of aqueous extracts obtained from leaves of Azadirachta indica, Trichilia pallens and Trichilia pallida and branches of Trichilia pallens was 11,54 $\%$, with no differences of the aqueous extracts obtained from branches of Pepper pseudocaryophyllus and Ocimum minimum and leaves of Ricinus communis and Ocimum minimum. According to the results, the different plant extracts had repellent activity on adults of banana weevil, nevertheless, the highest percentages were observed when using extract derived from seeds of Azadirachta indica and leaves of Pimenta pseudocaryophyllus.
\end{abstract}

Keywords: Botanical insecticides. Insect resistance. Pest management.

\footnotetext{
${ }^{1}$ Eng. Agr. Docente da UNESP - Campus Experimnetal de Registro.

${ }^{2}$ Alunos do curso de Agronomia da UNESP - Campus Experimental de Registro
} 


\section{INTRODUÇÃO}

No Brasil, a banana é cultivada em todos os Estados, desde a faixa litorânea até os planaltos interioranos, já que se constitui em meio de subsistência para as populações rurais, além de ser um produto de exportação. Embora o Brasil seja um dos maiores produtores mundiais de banana, exporta somente uma pequena parcela de sua produção, muito inferior, por exemplo, a de outros países como o Equador, cuja produção é menor que a brasileira, mas que exporta praticamente $65 \%$ de sua produção. Esta baixa representatividade do Brasil devese principalmente à baixa produtividade e ao alto índice de perdas, causadas principalmente por problemas fitossanitários (BATISTA FILHO; TAKADA; CARVALHO, 2002).

Dentre os problemas fitossanitários ressalta-se o Cosmopolites sordidus (Germar) (Coleoptera: Curculionidae) que é praga em quase todos os países produtores de banana, provocando perdas de 30 a 90\% (GOLD; PINESE; PEÑA, 2002). No Brasil, principalmente no estado de São Paulo, foi considerada a praga mais importante na década de 80 (SUPLICY FILHO; SAMPAIO, 1982). Segundo Gallo et al (2002), infestações de 12 larvas/planta podem afetar a produção entre 20 a 50\%. Os danos causados à bananeira ocorrem, principalmente, quando a praga se encontra na fase larval, quando produz galerias nos rizomas e na parte inferior do pseudocaule, afetando o desenvolvimento e a produção, além de causar a queda das plantas pela ação dos ventos (BATISTA FILHO; TAKADA; CARVALHO, 2002).

A broca tem preferência pelos cultivares Maçã e Terra. Nas plantações bem cuidadas de cultivares do subgrupo Cavendish, esse curculionídeo não é considerado causador de prejuízos significativos. Em bananais mal desbastados, mal desfolhados e sem controle do mato e da própria broca, essa praga pode causar prejuízos, chegando a anular completamente a produção (MOREIRA, 1999).

Inseticidas botânicos têm sido relatados por ter uma ampla gama de atividades biológicas contra insetos (BERENBAUM, 1989). Estes incluem repelência, alimentação ou oviposição, dissuasão, toxicidade, esterilidade e crescimento regulamentar (JACOBSON, 1989; SCHMUTTERER, 1995; ASCHER, 1993). O objetivo deste trabalho foi avaliar a porcentagem de repelência dos extratos aquosos de plantas com atividade inseticida sobre o adulto da broca-da-bananeira (C. sordidus).

\section{MATERIAL E MÉTODOS}

O presente trabalho foi realizado no laboratório da UNESP-Campus Experimental de Registro, utilizando-se delineamento em blocos casualizados, com dez tratamentos e cinco 
repetições, à temperatura de $25 \pm 2^{0} \mathrm{C}$, umidade relativa de $60 \pm 10 \%$ e fotofase de 14 horas. Os insetos utilizados no experimento foram coletados em bananal comercial localizado no município de Registro, SP, utilizando iscas de pseudocaule tipo "queijo".

Pedaços de pseudocaule coletados no bananal foram mantidos em laboratório acondicionados em balde plástico de 10 litros (24 cm de diâmetro e $26 \mathrm{~cm}$ de profundidade) servindo de alimento aos adultos durante a realização do experimento.

\section{Seleção de espécies de plantas e preparação dos extratos aquosos}

Seis espécies de plantas foram selecionadas para o estudo de sua atividade repelente sobre os adultos de Cosmopolites sordidus, sendo essas: Azadirachta indica, Trichilia pallida, Pimenta pseudocaryophyllus, Trichilia pallens, Ricinus communis e Ocimum minimum.

Para a obtenção dos extratos estruturas de cada planta foram secas em estufa de ar forçado a temperatura de $45^{\circ} \mathrm{C}$ e depois moídas, obtendo-se assim o extrato vegetal bruto. $\mathrm{Na}$ sequência pesou-se $20 \mathrm{~g}$ de cada extrato bruto em balança analítica, sendo transferidos para Beckers de $100 \mathrm{ml}$, completando cada recipiente com água destilada até $100 \mathrm{ml}$, mexendo-se por alguns minutos e depois colocando em um recipiente de vidro para descanso por $24 \mathrm{~h}$. Após este período, o material foi coado em tecido de malha fina, obtendo-se assim o extrato aquoso na concentração de $20 \%$ para realização dos testes.

Os tratamentos consistiram dos extratos aquosos de folhas e sementes de A. indica (neem), folhas de T. pallida (catiguá), folhas e ramos de T. pallens (baga de morcego), folha e ramos de P. pseudocaryophyllus (cataia), folhas de $R$. communis (mamona), folhas e ramos de O. minimum (alfavaca) e água destilada como controle (testemunha) em cinco repetições cada.

Utilizou-se um recipiente de alumínio ( $28 \mathrm{~cm}$ de diâmetro e $7 \mathrm{~cm}$ de profundidade) onde foram distribuídos dois pedaços de igual tamanho $(5 \times 5 \times 5 \mathrm{~cm})$ de pseudocaule em suas extremidades circundantes, um proveniente da imersão em um determinado extrato aquoso, e outro proveniente da imersão em água destilada, ambos durante sessenta segundos. $\mathrm{Na}$ sequência foram liberados dez insetos adultos no centro de cada recipiente. Após a montagem o recipiente foi fechado com tecido de malha fina (voal) na parte superior, obtendo-se assim um bloco do delineamento experimental. Foram montados cinquenta blocos no total do experimento e após $48 \mathrm{~h}$ foi feita a avaliação da preferência dos insetos adultos em cada bloco.

\section{Bioensaio de repelência}

Para obtenção dos dados sobre porcentagem de repelência foi estabelecido o modelo 
matemático proposto por Inyang e Emosairue (2005), sendo este:

$$
P R=\left(\frac{N C-T N}{N C+T N}\right) \times 100
$$

PR = Porcentagem de Repelência (\%)

$\mathrm{NC}=$ número de insetos presente no pseudocaule tratado com água destilada (controle)

$\mathrm{NT}=$ número de insetos presente no pseudocaule tratado com extrato aquoso da planta

$* \mathrm{PR}=$ os valores negativos deveram ser transformados para arcoseno.

Assim baseados nos cálculos obteve-se dados quanto à porcentagem de repelência dos diferentes extratos testados.

Os dados obtidos foram submetidos à análise de variância, com aplicação do teste $\mathrm{F}$ e posteriormente suas médias comparadas com o teste de Tukey, a 5\% de probabilidade.

\section{RESULTADOS E DISCUSSÃO}

Os maiores valores em relação a repelência foram observados quando se utilizou extrato obtido a partir de sementes de A. indica e folhas de P. pseudocaryophyllus, com 48 e $24 \%$ de repelência, respectivamente, sendo estes valores estatisticamente diferentes entre si (Tabela 1). A porcentagem de repelência dos extratos aquosos obtidos a partir de folhas de $A$. indica, T. pallens e T. pallida e ramos de $T$. pallens foi de 11,54 \%, não diferindo estatisticamente dos extratos aquosos obtidos a partir de ramos de P. pseudocaryophyllus e $O$. minimum $e$ folhas de $R$. communis e $O$. minimum. De acordo com a tabela 1 , a porcentagem de repelência do extrato aquoso obtido a partir de folhas de $O$. minimum foi de $4 \%$ sendo a metade da eficiência quando comparado aos extratos obtidos a partir de folhas de $R$. communis e ramos de O. minimum, com $8 \%$ (Figura 1).

Inyang e Emosairue (2005) testaram, em laboratório, o uso de extrato proveniente de sementes de $A$. indica na concentração de $5 \%$ e após 48 horas obtiveram $49 \%$ de repelência sobre adultos de $C$. sordidus. Este dado é próximo do observado nesse trabalho com o extrato aquoso obtido a partir de sementes de $A$. indica na concentração de $20 \%$. Estes mesmos autores obtiveram $60 \%$ de repelência de $C$. sordidus quando trabalharam com sementes de $A$. Indica na concentração de $10 \%$.

Estas variações nos resultados apresentados, quando comparados aos dados de literatura, podem ser devido a outros fatores relacionados ao inseto ou a planta utilizada como alimento. No entanto, percebe-se que sementes de $A$. indica apresentam considerável efeito de repelência sobre adultos deste inseto, podendo ser uma ferramenta adicional ao seu manejo. 
Tabela 1. Porcentagem de repelência de diferentes extratos aquosos de plantas sobre adultos da broca-da-bananeira (Cosmopolites sordidus).

\section{Extratos de plantas}

Porcentagem de Repelência após 48

h

Azadirachta indica (folha)

$11,54 \pm 0,25^{\mathrm{c}}$

Azadirachta indica (semente)

$48,00 \pm 0,44^{\mathrm{a}}$

Pimenta pseudocaryophyllus (folha)

$24,00 \pm 0,33^{\mathrm{b}}$

Pimenta pseudocaryophyllus (ramo)

$9,20 \pm 0,32^{\mathrm{c}}$

Trichilia pallens (folha)

$11,54 \pm 0,30^{c}$

Trichilia pallens (ramo)

$11,54 \pm 0,41^{\mathrm{c}}$

Trichilia pallida (folha)

$11,54 \pm 0,39^{c}$

Ricinus communis (folha)

$8,00 \pm 0,20^{\mathrm{c}}$

Ocimum minimum (folha)

$4,00 \pm 0,25^{\mathrm{c}}$

Ocimum minimum (ramo)

$8,00 \pm 0,23^{\mathrm{c}}$

* Médias seguidas pela mesma letra na coluna não diferem entre si, pelo teste de Tukey a 5\% de probabilidade.

$*$ Valores $\pm \mathrm{SE}$

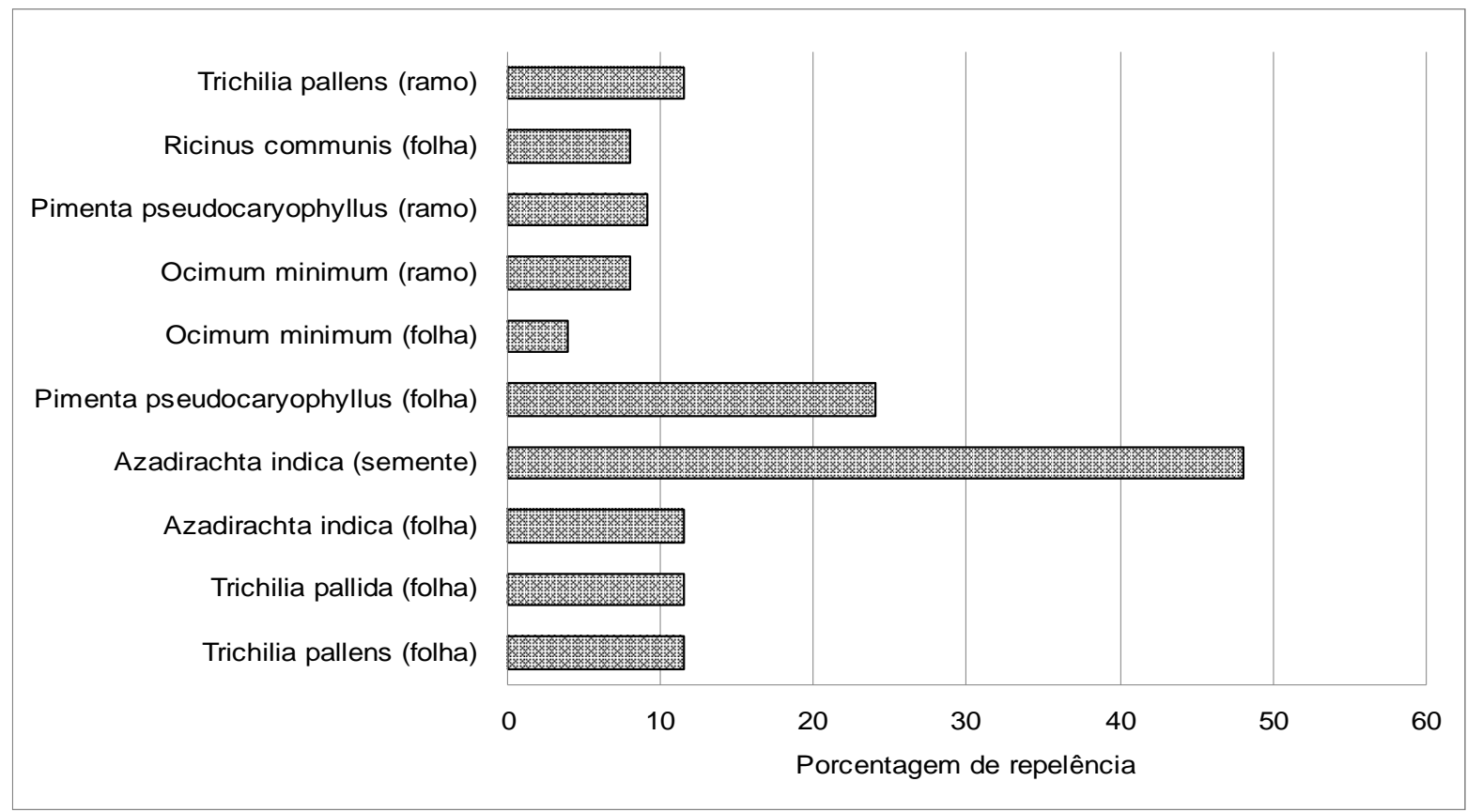

Figura 1. Porcentagem de repelência de diferentes extratos aquosos de plantas sobre adultos da broca-da-bananeira (C. sordidus).

\section{CONCLUSÃO}

De acordo com os resultados obtidos, conclui-se que os diferentes extratos aquosos das plantas testadas apresentaram atividade de repelência sobre adultos da broca-da-bananeira 
(Cosmopolites sordidus), sendo as maiores obtidas pelo uso dos extratos de semente de Azadirachta indica e de folha de Pimenta pseudocaryophyllus.

\section{REFERÊNCIAS}

ASCHER, K. R. S. Nonconventional insecticidal effects of pesticides available from the neem tree, Azadirachta indica. Arch.Insect Bioch Phys. 22, p.433-449, 1993.

BATISTA FILHO, A.; TAKADA, H. M.; CARVALHO, A.G. Brocas da bananeira. In: REUNIÃO ITINERANTE DE FITOSSANIDADE DO INSTITUTO BIOLÓGICO BANANA, 6., 2002, São Bento do Sapucaí - SP, 2002. Anais... São Paulo: Instituto Biológico, 2002. p.1-16.

BERENBAUM, R. M. North America ethnobotanicals as sources of novel plant based insecticides. In: Arnason JJ, Philogene, BR, Morand P (eds) Insecticides of plant origin, ACS Sympos. Ser. 387, p.11-24, 1989.

GOLD, C. S.; PINESE, B.; PEÑA, J E. Pests of Banana. In: PEÑA, J. E. (Ed.) Tropical fruit pests and pollinators: biology, economic importance, natural enemies and control. Florida: Cabi Publishing, cap.2, p.13-32, 2002.

GALLO, D.et al. Entomologia agrícola. São Paulo: Agronômica Ceres, 2002.

INYANG, U. E. ; EMOSAIRUE, S. O. Laboratory assessment of the repellent and antifeedant properties of aqueous extracts of 13 plants against the banana weevil Cosmopolites sordidus Germar (Coleoptera: Curculionidae) Tropical and Subtropical Agroecosystems, v. 5, p. $33-44,2005$.

JACOBSON, M. Botanical pesticides, past, present and future. In: Arnason JJ, Philogene BR, Morand P (eds) Insecticides of plant origin, ACS Symposium Ser. 387, p. 1-10, 1989.

MOREIRA, R. S. Banana, teoria e prática de cultivo. 2. ed. Fundação Cargill. 1999.

SCHMUTTERER, H. The neem tree Azadirachta indica A. Juss and other Maliaceous plant sources of unique natural products of integrated pest management medicine, industry and other purposes. VCH, 1995.

SUPLICY FILHO, N.; SAMPAIO, A. S. Pragas da bananeira. Biológico, São Paulo, v.48, n.7, p.169-182, 1982. 